Article

\title{
Total Syntheses of Telisatin A, Telisatin B and Lettowianthine
}

\section{Surachai Nimgirawath* and Phansuang Udomputtimekakul}

Department of Chemistry, Faculty of Science, Silpakorn University; Nakorn Pathom 73000, Thailand; E-mail: Phansuang@yahoo.com (P.U.)

* Author to whom correspondence should be addressed; E-mail: surachai@su.ac.th.

Received: 2 February 2009; in revised form: 17 February 2009/ Accepted: 26 February 2009 / Published: 26 February 2009

\begin{abstract}
Treatment of 1-(2-bromoarylmethyl)-3,4-dihydroisoquinolines with oxalyl chloride and triethylamine gave 1-(2-bromophenyl)-5,6-dihydropyrrolo[2,1-a]isoquinoline2,3-dione derivatives, for example, 1-(2-bromophenyl)-5,6-dihydro-8,9dimethoxypyrrolo[2,1-a]isoquinoline-2,3-dione. Radical cyclisation of these derivatives with tributyltin hydride and 1,1'-azobis(cyclohexanecarbonitrile) afforded telisatin A, telisatin B and lettowianthine.
\end{abstract}

Keywords: Alkaloid; Dioxoaporphine; Isoquinoline; Radical cyclisation; Synthesis.

\section{Introduction}

The telisatin-type aporphine alkaloids form a very small sub-group of the aporphine alkaloids in which N-6 and C-7 are fused to an oxalyl function. To date only five members of this type of aporphine alkaloids have been found to occur in Nature. These are telisatin A (1) and telisatin B (2) from Telitoxicum peruvianum Moldenke (Menispermaceae) [1], lettowianthine (3) and 11-methoxylettowianthine (4) from Lettowianthus stellatus Diels (Annonaceae) [2], and laurodionine (5) from Phoebe formosana Hayata (Lauraceae) [3]. Annonbraine, isolated from Annona glaba L (Annonaceae), was also assigned the same structure as lettowianthine (3), although there was a big difference in the melting points of the two alkaloids [4]. The structure of telisatin A was elucidated by comparison of spectral data and physical properties with a synthetic compound obtained by Saa and 
Cava [5], Castedo et al. [6] and Saa et al. [7]. The structures of the remaining alkaloids were assigned based on spectral data analysis.

Figure 1. Structures of telisatin A (1), telisatin B (2), lettowianthine (annonbraine) (3), 11-methoxylettowianthine (4) and laurodionine (5).

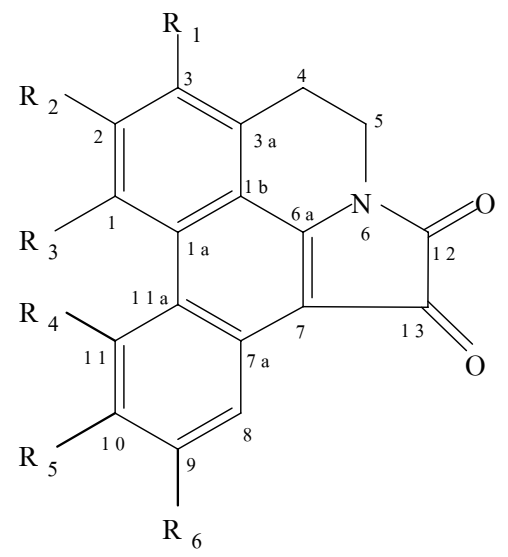

$$
\begin{aligned}
& 1: \mathrm{R}_{1}=\mathrm{R}_{4}=\mathrm{R}_{5}=\mathrm{R}_{6}=\mathrm{H}, \mathrm{R}_{2}=\mathrm{R}_{3}=\mathrm{OCH}_{3} \text { (telisatin } \mathrm{A} \text { ) } \\
& 2: \mathrm{R}_{1}=\mathrm{R}_{2}=\mathrm{R}_{3}=\mathrm{OCH}_{3}, \mathrm{R}_{4}=\mathrm{R}_{5}=\mathrm{R}_{6}=\mathrm{H} \text { (telisatin } \mathrm{B} \text { ) } \\
& 3: \mathrm{R}_{1}=\mathrm{R}_{4}=\mathrm{R}_{5}=\mathrm{R}_{6}=\mathrm{H}, \mathrm{R}_{2}+\mathrm{R}_{3}=\mathrm{OCH}_{2} \mathrm{O} \text { (lettowianthine, annonbraine) } \\
& 4: \mathrm{R}_{1}=\mathrm{R}_{5}=\mathrm{R}_{6}=\mathrm{H}, \mathrm{R}_{2}+\mathrm{R}_{3}=\mathrm{OCH}_{2} \mathrm{O}, \mathrm{R}_{4}=\mathrm{OCH}_{3}(11 \text {-methoxylettowianthine) } \\
& 5: \mathrm{R}_{1}=\mathrm{R}_{4}=\mathrm{H}, \mathrm{R}_{2}=\mathrm{R}_{6}=\mathrm{OH}, \mathrm{R}_{3}=\mathrm{R}_{5}=\mathrm{OCH}_{3} \text { (laurodionine) }
\end{aligned}
$$

At present there are only two total syntheses of telisatin A reported by Castedo et al. [6] and by Saa et al. [7] The first method involved photochemical cyclisation in reasonably good yield (60\%) of a very dilute $(0.001 \mathrm{M})$ solution of 1-(2-bromophenyl)-5,6-dihydro-8,9-dimethoxypyrrolo[2,1a]isoquinoline-2,3-dione (10a) [6]. This method has obvious inherent limitations with regards to its scalability and convenience. The second method, based on benzyne cycloaddition, gave a low yield (10\%) [7]. A partial synthesis reported by Saa and Cava involved acylation of 6a,7-dehydronuciferine with oxalyl chloride [5]. Since 6a,7-dehydroaporphines themselves are not readily accessible, this method therefore lacks generality and convenience.

\section{Results and Discussion}

We would like to report herein an extension of the first method described by Castedo et al. [6], shown in Scheme 1. Amides 8a-8c, obtained by conventional methods, were converted by a BischlerNapieralski reaction to dihydroisoquinolines 9a-9c. We found that 1-(2-bromophenyl)-5,6dihydropyrrolo[2,1-a]-isoquinoline-2,3-dione derivatives 10a-10c could be more conveniently prepared by the reaction of dihydroisoquinolines 9a-9c with oxalyl chloride in the presence of triethylamine with straightforward workup [8]. The antiplatelet activity of such 1-aryl-5,6dihydropyrrolo[2,1-a]-isoquinoline-2,3-dione derivatives has been reported [9]. To overcome the limitations of photochemical cyclisation under extreme dilution and based on previous reports on the radical cyclisation of halostilbenes to phenanthrenes [10-14], solutions (0.025 M) of 1-(2- 
bromophenyl)-5,6-dihydropyrrolo[2,1-a]isoquinoline-2,3-dione derivatives 10a-10c were treated with tributyltin hydride in the presence of $1,1^{\prime}$-azobis(cyclohexanecarbonitrile) (ACCN) to give the corresponding telisatin-type alkaloids in $30-34 \%$ yields.

Scheme 1. Syntheses of telisatin A (1), telisatin B (2) and lettowianthine (3).<smiles>[R4]c1ccc(CCN)c([R])c1[R4]</smiles>

$6 \mathrm{a}, 6 \mathrm{~b}, 6 \mathrm{c}$<smiles>O=C(Cl)Cc1ccccc1Br</smiles><smiles>[R3]c1ccc(CCNC(=O)Cc2ccccc2Br)c([R])c1[R4]</smiles>

$8 \mathrm{a}, 8 \mathrm{~b}, 8 \mathrm{c}$<smiles>[R]c1cc2c(c([R])c1[R])CCN=C2Cc1ccccc1Br</smiles>

$9 \mathbf{a}, 9 \mathbf{b}, 9 \mathrm{c}$<smiles>[R]Cc1cc2c(c([R])c1[R4])CCN1C(=O)C(=O)C(c3ccccc3Br)=C21</smiles>

$10 \mathrm{a}, 10 \mathrm{~b}, 10 \mathrm{c}$<smiles></smiles>

$1,2,3$

a : $\mathrm{R}_{1}=\mathrm{H}, \mathrm{R}_{2}=\mathrm{R}_{3}=\mathrm{OCH}_{3}$

b : $\mathrm{R}_{1}=\mathrm{R}_{2}=\mathrm{R}_{3}=\mathrm{OCH}_{3}$

c : $\mathrm{R}_{1}=\mathrm{H}, \mathrm{R}_{2}+\mathrm{R}_{3}=\mathrm{OCH}_{2} \mathrm{O}$

Reaction conditions: A) $10 \% \mathrm{NaHCO}_{3} /$ chloroform; B) $\mathrm{POCl}_{3} /$ benzene; C) oxalyl chloride, triethylamine/ chloroform; D) $\mathrm{Bu}_{3} \mathrm{SnH}, \mathrm{ACCN} /$ dry toluene.

Fortunately, in all cases, it was possible to isolate the pure alkaloids from the crude reaction mixtures by simple crystallization from ethanol. Silica gel chromatography of the residues from the filtrates afforded only minute quantities of the pure alkaloids and was therefore not pursued any further. Comparison of the yields from radical cyclisation using ACCN with those obtained using 2,2'azobis(isobutyronitrile)(AIBN) was not possible since AIBN is no longer commercially available in Thailand. The spectral data of synthetic telisatin A (1), telisatin B (2) and lettowianthine (3) were in good agreement to those reported for the natural alkaloids.

\section{Conclusions}

We have developed an easy and convenient synthesis of the telisatin-type alkaloids. Further applications of the current synthesis to the remaining telisatin-type alkaloids are in progress. 


\section{Experimental}

\section{General}

Melting points were determined on a Stuart Scientific SMP 2 melting point apparatus and are uncorrected. Infrared spectra were recorded on $\mathrm{CH}_{2} \mathrm{Cl}_{2}$-films with a Perkin Elmer Spectrum GX FT-IR spectrophotometer. Ultraviolet spectra were recorded on methanol solutions with a Hitachi U-3300 spectrophotometer. ${ }^{1} \mathrm{H}$ - and ${ }^{13} \mathrm{C}$-NMR spectra were recorded for deuterochloroform solutions at 300 $\mathrm{MHz}$ for ${ }^{1} \mathrm{H}$ and $75 \mathrm{MHz}$ for ${ }^{13} \mathrm{C}$ with a Bruker AVANCE 300 spectrometer. Tetramethylsilane was used as the internal standard. High Resolution Mass spectra were recorded with a Bruker Daltonics MicrOTOF mass spectrometer.

$N$-(3,4-Dimethoxyphenethyl)-2-(2-bromophenyl)acetamide (8a). A mixture of 2-bromophenylacetic acid (15.0 g, $0.07 \mathrm{~mol})$ and thionyl chloride $(20.8 \mathrm{~g})$ in benzene $(50 \mathrm{~mL})$ was refluxed for $1 \mathrm{~h}$. Removal of the solvent under vacuum gave 2-bromophenylacetyl chloride (7) which was dissolved in ethanol-free chloroform $(50 \mathrm{~mL})$ and added to a mixture of 3,4-dimethoxyphenethylamine $(\mathbf{6 a}, 12.7 \mathrm{~g}$, $0.07 \mathrm{~mol})$ in chloroform $(100 \mathrm{~mL})$ and $10 \%$ sodium hydrogen carbonate $(100 \mathrm{~mL})$. The mixture was then stirred for $3 \mathrm{~h}$ and the chloroform layer was washed with water $(2 \times 100 \mathrm{~mL}), 10 \%$ hydrochloric acid $(3 \times 50 \mathrm{~mL})$, water $(100 \mathrm{~mL})$, then dried over anhydrous sodium sulfate. Removal of the solvent under vacuum gave a residue which was recrystallized from ethanol to give amide 8a as a pale yellow solid (22.0 g, 88.1\%); m.p. 131-132 ${ }^{\circ} \mathrm{C}$ (Lit. [15] m.p. 127-129 $\left.{ }^{\circ} \mathrm{C}\right) ;{ }^{1} \mathrm{H}-\mathrm{NMR}: \delta 7.54(1 \mathrm{H}, \mathrm{d}, J=7.8$ $\mathrm{Hz}, \mathrm{Ar}-\mathrm{H}) ; 7.30-7.22$ (2H, m, Ar-H); 7.18-7.09 (1H, m, Ar-H); $6.72(1 \mathrm{H}, \mathrm{d}, J=8.1 \mathrm{~Hz}, \mathrm{Ar}-\mathrm{H}) ; 6.63$ $(1 \mathrm{H}, \mathrm{d}, J=1.9 \mathrm{~Hz}, \mathrm{Ar}-\mathrm{H}) ; 6.60(1 \mathrm{H}, \mathrm{dd}, J=8.1,1.9 \mathrm{~Hz}, \mathrm{Ar}-\mathrm{H}) ; 5.58(1 \mathrm{H}, \mathrm{s}, \mathrm{NH}) ; 3.85\left(3 \mathrm{H}, \mathrm{s}, \mathrm{OCH}_{3}\right)$; $3.82\left(3 \mathrm{H}, \mathrm{s}, \mathrm{OCH}_{3}\right) ; 3.66\left(2 \mathrm{H}, \mathrm{s}, \mathrm{CH}_{2}\right) ; 3.47\left(2 \mathrm{H}\right.$, apparent q, $\left.J=6.8 \mathrm{~Hz}, \mathrm{CH}_{2}\right) ; 2.71(2 \mathrm{H}$, apparent t, $J$ $\left.=7.0 \mathrm{~Hz}, \mathrm{CH}_{2}\right)$; ${ }^{13} \mathrm{C}-\mathrm{NMR}: \delta 169.48(\mathrm{C}), 148.98(\mathrm{C}), 147.60(\mathrm{C}), 134.79(\mathrm{C}), 133.03(\mathrm{CH}), 131.63(\mathrm{CH})$, 131.10(C), 129.04(CH), 127.92(CH), 124.94(C), 120.58(CH), 111.79(CH), 111.32(CH), 55.91 $\left(\mathrm{OCH}_{3}\right)$, $55.82\left(\mathrm{OCH}_{3}\right), 43.99\left(\mathrm{CH}_{2}\right), 40.81\left(\mathrm{CH}_{2}\right), 34.99\left(\mathrm{CH}_{2}\right)$.

$N$-(2,3,4-Trimethoxyphenethyl)-2-(2-bromophenyl)acetamide (8b). In a similar manner, 8b was obtained in $78.8 \%$ yield as a pale yellow solid from ethanol; m.p. 92-93 ${ }^{\circ} \mathrm{C} ;{ }^{1} \mathrm{H}-\mathrm{NMR}: \delta 7.57(1 \mathrm{H}, \mathrm{d}, J$ $=8.3 \mathrm{~Hz}, \mathrm{Ar}-\mathrm{H}) ; 7.33-7.25(2 \mathrm{H}, \mathrm{m}, \mathrm{Ar}-\mathrm{H}) ; 7.19-7.12(1 \mathrm{H}, \mathrm{m}, \mathrm{Ar}-\mathrm{H}) ; 6.72(1 \mathrm{H}, \mathrm{d}, J=8.5 \mathrm{~Hz}, \mathrm{Ar}-\mathrm{H})$; $6.54(1 \mathrm{H}, \mathrm{d}, J=8.5 \mathrm{~Hz}, \mathrm{Ar}-\mathrm{H}) ; 5.83(1 \mathrm{H}, \mathrm{br} \mathrm{s}, \mathrm{NH}) ; 3.88\left(3 \mathrm{H}, \mathrm{s}, \mathrm{OCH}_{3}\right) ; 3.83\left(3 \mathrm{H}, \mathrm{s}, \mathrm{OCH}_{3}\right) ; 3.79$ $\left(3 \mathrm{H}, \mathrm{s}, \mathrm{OCH}_{3}\right) ; 3.67\left(2 \mathrm{H}, \mathrm{s}, \mathrm{CH}_{2}\right) ; 3.43\left(2 \mathrm{H}\right.$, apparent q, $\left.J=6.4 \mathrm{~Hz}, \mathrm{CH}_{2}\right) ; 2.70(2 \mathrm{H}$, apparent $\mathrm{t}, J=6.7$ $\left.\mathrm{Hz}, \mathrm{CH}_{2}\right) ;{ }^{13} \mathrm{C}-\mathrm{NMR}: \delta 169.58(\mathrm{C}), 152.59(\mathrm{C}), 151.74(\mathrm{C}), 142.19(\mathrm{C}), 134.91(\mathrm{C}), 133.05(\mathrm{CH})$, 131.78(CH), 128.97(CH), 127.89(CH), 125.10(C), 124.69(C), 124.46(CH), 107.42(CH), 60.92( $\left.\mathrm{OCH}_{3}\right)$, 60.75( (OCH 3$), 56.01\left(\mathrm{OCH}_{3}\right), 44.05\left(\mathrm{CH}_{2}\right), 40.91\left(\mathrm{CH}_{2}\right), 29.47\left(\mathrm{CH}_{2}\right)$.

$\mathrm{N}$-(3,4-Methylenedioxyphenethyl)-2-(2-bromophenyl)acetamide (8c). In a similar manner, 8c was obtained in $82.5 \%$ yield as a pale yellow solid from ethanol; m.p. $124-126{ }^{\circ} \mathrm{C}$ (Lit. [16] m.p. 128-130 $\left.{ }^{\circ} \mathrm{C}\right)$; ${ }^{1} \mathrm{H}-\mathrm{NMR}: \delta 7.56(1 \mathrm{H}, \mathrm{d}, J=7.8 \mathrm{~Hz}, \operatorname{Ar}-\mathrm{H}) ; 7.33-7.25(2 \mathrm{H}, \mathrm{m}, \operatorname{Ar}-\mathrm{H}) ; 7.19-7.11(1 \mathrm{H}, \mathrm{m}, \operatorname{Ar}-\mathrm{H})$; $6.65(1 \mathrm{H}, \mathrm{d}, J=7.9 \mathrm{~Hz}, \mathrm{Ar}-\mathrm{H}) ; 6.55(1 \mathrm{H}, \mathrm{d}, J=1.6 \mathrm{~Hz}, \mathrm{Ar}-\mathrm{H}) ; 6.48(1 \mathrm{H}, \mathrm{dd}, J=7.9,1.6 \mathrm{~Hz}, \mathrm{Ar}-\mathrm{H})$; $5.91\left(2 \mathrm{H}, \mathrm{s}, \mathrm{OCH}_{2} \mathrm{O}\right) ; 5.48(1 \mathrm{H}$, br s, $\mathrm{NH}) ; 3.66\left(2 \mathrm{H}, \mathrm{s}, \mathrm{CH}_{2}\right) ; 3.42\left(2 \mathrm{H}\right.$, apparent q, $\left.J=6.7 \mathrm{~Hz}, \mathrm{CH}_{2}\right)$; 
$2.65\left(2 \mathrm{H}\right.$, apparent t, $\left.J=6.7 \mathrm{~Hz}, \mathrm{CH}_{2}\right) ;{ }^{13} \mathrm{C}-\mathrm{NMR}: \delta 169.44(\mathrm{C}), 147.72(\mathrm{C}), 146.11(\mathrm{C}), 134.80(\mathrm{C})$, 133.13(CH), 132.35(C), 131.67(CH), 129.09(CH), 127.97(CH), 124.98(C), 121.57(CH), 109.04(CH), 108.30(CH), 100.85( $\left(\mathrm{CH}_{2}\right), 44.04\left(\mathrm{CH}_{2}\right), 40.87\left(\mathrm{CH}_{2}\right), 35.15\left(\mathrm{CH}_{2}\right)$.

1-(2-Bromobenzyl)-3,4-dihydro-6,7-dimethoxyisoquinoline (9a). A solution of 8a (5.5 g, $14.6 \mathrm{mmol})$ and phosphorus oxychloride $(17.0 \mathrm{~g})$ in benzene $(60 \mathrm{~mL})$ was refluxed for $3 \mathrm{~h}$. The excess reagent and solvent were removed under vacuum. The residue was shaken with chloroform $(100 \mathrm{~mL})$ and dilute ammonium hydroxide $(100 \mathrm{~mL})$. The chloroform layer was washed with water $(100 \mathrm{~mL})$, then dried over anhydrous sodium carbonate. Removal of the solvent under vacuum gave dihydroisoquinoline 9a as a pale yellow solid (3.9 g, 75.8\%) from ethyl acetate-hexane; m.p. 95-96 ${ }^{\circ} \mathrm{C}$ (Lit. [15] m.p. 93-95 $\left.{ }^{\circ} \mathrm{C}\right)$. It was found to be unstable and was immediately used in the next step without further purification. ${ }^{1} \mathrm{H}-\mathrm{NMR}: \delta 7.56(1 \mathrm{H}, \mathrm{dd}, J=7.9,1.3 \mathrm{~Hz}, \mathrm{Ar}-\mathrm{H}) ; 7.27(1 \mathrm{H}, \mathrm{dd}, J=7.6,1.7 \mathrm{~Hz}, \mathrm{Ar}-\mathrm{H}) ; 7.18(1 \mathrm{H}, \mathrm{dt}, J$ $=7.6,1.3 \mathrm{~Hz}, \mathrm{Ar}-\mathrm{H}) ; 7.05(1 \mathrm{H}, \mathrm{dt}, J=7.6,1.7 \mathrm{~Hz}, \mathrm{Ar}-\mathrm{H}) ; 6.91(1 \mathrm{H}, \mathrm{s}, \mathrm{Ar}-\mathrm{H}) ; 6.67(1 \mathrm{H}, \mathrm{s}, \mathrm{Ar}-\mathrm{H}) ; 4.20$ $\left(2 \mathrm{H}, \mathrm{s}, \mathrm{Ar}-\mathrm{CH}_{2}\right) ; 3.88\left(3 \mathrm{H}, \mathrm{s}, \mathrm{OCH}_{3}\right) ; 3.79\left(3 \mathrm{H}, \mathrm{s}, \mathrm{OCH}_{3}\right) ; 3.73\left(2 \mathrm{H}, \mathrm{t}, J=7.6 \mathrm{~Hz}, \mathrm{CH}_{2}\right) ; 2.67(2 \mathrm{H}, \mathrm{t}, J$ $\left.=7.6 \mathrm{~Hz}, \mathrm{CH}_{2}\right) ;{ }^{13} \mathrm{C}-\mathrm{NMR} \delta: 165.08(\mathrm{C}), 150.76(\mathrm{C}), 147.43(\mathrm{C}), 137.66(\mathrm{C}), 132.78(\mathrm{CH}), 131.59(\mathrm{C})$, $130.21(\mathrm{CH}), \quad 128.18(\mathrm{CH}), \quad 127.62(\mathrm{CH}), \quad 124.54(\mathrm{C}), \quad 121.41(\mathrm{C}), \quad 110.25(\mathrm{CH}), \quad 109.15(\mathrm{CH})$, 56.16( $\left(\mathrm{OCH}_{3}\right)$, 55.92( $\left(\mathrm{OCH}_{3}\right), 47.29\left(\mathrm{CH}_{2}\right), 42.53\left(\mathrm{CH}_{2}\right), 25.73\left(\mathrm{CH}_{2}\right)$.

1-(2-Bromobenzyl)-3,4-dihydro-5,6,7-trimethoxyisoquinoline (9b). In a similar manner, 9b was obtained in almost quantitative yield as a yellow viscous oil. ${ }^{1} \mathrm{H}-\mathrm{NMR}: \delta 7.55(1 \mathrm{H}, \mathrm{dd}, J=7.9,1.2 \mathrm{~Hz}$, Ar-H); 7.30-7.26 (1H, m, Ar-H); 7.20-7.15 (1H, m, Ar-H); 7.09-7.01 (1H, m, Ar-H); $6.78(1 \mathrm{H}, \mathrm{s}$, $\mathrm{H}-8) ; 4.19\left(2 \mathrm{H}, \mathrm{s}, \mathrm{Ar}-\mathrm{CH}_{2}\right) ; 3.88\left(3 \mathrm{H}, \mathrm{s}, \mathrm{OCH}_{3}\right) ; 3.83\left(3 \mathrm{H}, \mathrm{s}, \mathrm{OCH}_{3}\right) ; 3.78\left(3 \mathrm{H}, \mathrm{s}, \mathrm{OCH}_{3}\right) ; 3.71(2 \mathrm{H}, \mathrm{t}$, $\left.J=7.6 \mathrm{~Hz}, \mathrm{CH}_{2}\right) ; 2.67\left(2 \mathrm{H}, \mathrm{t}, J=7.6 \mathrm{~Hz}, \mathrm{CH}_{2}\right) ;{ }^{13} \mathrm{C}-\mathrm{NMR}: \delta 164.68(\mathrm{C}), 151.69(\mathrm{C}), 149.88(\mathrm{C}), 144.17$ (C), 137.63(C), 132.77(CH), 130.23(CH), 128.19(CH), 127.61(CH), 124.52(C), 124.46(C), 124.09(C), 105.63(CH), 60.88( $\left(\mathrm{OCH}_{3}\right), 60.83\left(\mathrm{OCH}_{3}\right), 56.19\left(\mathrm{OCH}_{3}\right), 47.09\left(\mathrm{CH}_{2}\right), 42.58\left(\mathrm{CH}_{2}\right), 18.99\left(\mathrm{CH}_{2}\right)$.

1-(2-Bromobenzyl)-3,4-dihydro-6,7-methylenedioxyisoquinoline (9c). In a similar manner, 9c was obtained in $42.1 \%$ yield from ethanol as a pale yellow solid; m.p. $122-123{ }^{\circ} \mathrm{C}$ (Lit. [16] m.p. 121-123 $\left.{ }^{\circ} \mathrm{C}\right)$; ${ }^{1} \mathrm{H}-\mathrm{NMR}: \delta 7.52(1 \mathrm{H}, \mathrm{dd}, J=7.8,1.0 \mathrm{~Hz}, \mathrm{Ar}-\mathrm{H}) ; 7.22-7.12(2 \mathrm{H}, \mathrm{m}, \mathrm{Ar}-\mathrm{H})$; 7.06- 6.98 (1H, m, Ar$\mathrm{H}) ; 6.89(1 \mathrm{H}, \mathrm{s}, \mathrm{Ar}-\mathrm{H}) ; 6.61(1 \mathrm{H}, \mathrm{s}, \mathrm{Ar}-\mathrm{H}) ; 5.86\left(2 \mathrm{H}, \mathrm{s}, \mathrm{OCH}_{2} \mathrm{O}\right) ; 4.09\left(2 \mathrm{H}, \mathrm{s}, \mathrm{Ar}_{-} \mathrm{CH}_{2}\right) ; 3.65(2 \mathrm{H}, \mathrm{t}, J$ $\left.=7.6 \mathrm{~Hz}, \mathrm{CH}_{2}\right) ; 2.60\left(2 \mathrm{H}, \mathrm{t}, J=7.6 \mathrm{~Hz}, \mathrm{CH}_{2}\right) ;{ }^{13} \mathrm{C}-\mathrm{NMR}: \delta 164.65(\mathrm{C}), 149.08(\mathrm{C}), 146.44(\mathrm{C})$, 137.55(C), 133.38(C), $132.79(\mathrm{CH}), 130.30(\mathrm{CH}), 128.14(\mathrm{CH}), 127.50(\mathrm{CH}), 124.79(\mathrm{C}), 122.77(\mathrm{C})$, 107.98(CH), 106.02(CH), 101.31( $\left(\mathrm{CH}_{2}\right), 47.09\left(\mathrm{CH}_{2}\right), 42.55\left(\mathrm{CH}_{2}\right), 26.33\left(\mathrm{CH}_{2}\right)$.

1-(2-Bromophenyl)-5,6-dihydro-8,9-dimethoxypyrrolo[2,1-a]isoquinoline-2,3-dione (10a). Oxalyl chloride $(0.2 \mathrm{~mL})$ was added dropwise to a stirred solution of 9 a $(359 \mathrm{mg}, 1 \mathrm{mmol})$, triethylamine $(0.3$ $\mathrm{mL})$ in chloroform $(10 \mathrm{~mL})$ at room temperature. Stirring was continued for $3 \mathrm{~h}$. Chloroform $(20 \mathrm{~mL})$ was added and the chloroform layer was washed with $5 \%$ hydrochloric acid $(4 \times 50 \mathrm{~mL})$, water $(50$ $\mathrm{mL}$ ), then dried over anhydrous sodium sulfate. Removal of the solvent under vacuum gave a residue which was recrystallized from ethanol to give 10a as red prisms $(247.8 \mathrm{mg}, 60.0 \%)$; m.p. $195-196{ }^{\circ} \mathrm{C}$. (lit. [6] m.p. 176-178 ${ }^{\circ} \mathrm{C}$ ); UV $\lambda_{\max }(\mathrm{MeOH}) \mathrm{nm}(\log \varepsilon): 203$ (4.36), 226sh (4.12), 262 (3.76), 322 
(3.65). IR ( $\mathrm{CH}_{2} \mathrm{Cl}_{2}$-film) $v_{\max } \mathrm{cm}^{-1}: 2937,2843,1744,1699,1594,1575,1515,1472,1428,1398$, 1337, 1312, 1291, 1270, 1225, 1187, 1101, 1034, 987, 865, 798, 735, 683; ${ }^{1} \mathrm{H}-\mathrm{NMR}: \delta 7.70$ (1H, dd, $J$ = 8.0, 0.9 Hz, Ar-H); 7.44-7.37 (1H, m, Ar-H); 7.34-7.29 (1H, m, Ar-H); 7.29-7.22 (1H, m, Ar-H); $6.77(1 \mathrm{H}, \mathrm{s}, \mathrm{Ar}-\mathrm{H}) ; 6.66(1 \mathrm{H}, \mathrm{s}, \mathrm{Ar}-\mathrm{H}) ; 3.95\left(3 \mathrm{H}, \mathrm{s}, \mathrm{OCH}_{3}\right) ; 3.89\left(2 \mathrm{H}, \mathrm{t}, J=6.3 \mathrm{~Hz}, \mathrm{CH}_{2}\right) ; 3.29(3 \mathrm{H}, \mathrm{s}$, $\left.\mathrm{OCH}_{3}\right) ; 3.10\left(2 \mathrm{H}, \mathrm{t}, J=6.3 \mathrm{~Hz}, \mathrm{CH}_{2}\right) ;{ }^{13} \mathrm{C}-\mathrm{NMR}: \delta 181.59(\mathrm{C}), 158.50(\mathrm{C}), 158.35(\mathrm{C}), 153.80(\mathrm{C})$, 148.14(C), 133.17(CH), 133.09(C), 132.83(CH), 132.41(C), 129.96(CH), 128.06(CH), 125.82(C), 116.68(C), 111.29(CH), 107.80(C), 56.27( $\left(\mathrm{OCH}_{3}\right)$, 55.17( $\left(\mathrm{OCH}_{3}\right), 36.37\left(\mathrm{CH}_{2}\right), 28.37\left(\mathrm{CH}_{2}\right) . \mathrm{HRMS}$ (ESI-TOF) calcd for $\mathrm{C}_{20} \mathrm{H}_{16} \mathrm{BrNO}_{4}\left(\left[\mathrm{M}+\mathrm{H}^{+}\right]\right)=414.0335$, Found 414.0438.

1-(2-Bromophenyl)-5,6-dihydro-7,8,9-trimethoxypyrrolo[2,1-a] isoquinoline-2,3-dione (10b). In a similar manner, 10b was obtained as a deep red solid in $68.2 \%$ yield after chromatography over alumina using dichloromethane as eluent; m.p. $69-70{ }^{\circ} \mathrm{C}$; $\mathrm{UV}(\mathrm{MeOH}) \lambda_{\max } \mathrm{nm}(\log \varepsilon): 203$ (4.66), 226sh (4.38), 258 (3.95), 332 (3.93); IR ( $\mathrm{CH}_{2} \mathrm{Cl}_{2}$-film) $v_{\max } \mathrm{cm}^{-1}$ : 2939, 2837, 1746, 1702, 1592, 1576, 1467, 1425, 1397, 1342, 1298, 1248, 1182, 1109, 1024, 986, 939, 914, 845, 752; ${ }^{1} \mathrm{H}-\mathrm{NMR}: \delta 7.71(1 \mathrm{H}$, dd, $J=8.0,0.9 \mathrm{~Hz}, \mathrm{Ar}-\mathrm{H}) ; 7.44-7.35$ (1H, m, Ar-H); 7.32-7.23 (2H, m, Ar-H); 6.53 (1H, s, Ar-H); 3.94 $\left(3 \mathrm{H}, \mathrm{s}, \mathrm{OCH}_{3}\right) ; 3.88\left(3 \mathrm{H}, \mathrm{s}, \mathrm{OCH}_{3}\right) ; 3.93-3.73\left(2 \mathrm{H}, \mathrm{m}, \mathrm{CH}_{2}\right) ; 3.27\left(3 \mathrm{H}, \mathrm{s}, \mathrm{OCH}_{3}\right) ; 3.20-3.00(2 \mathrm{H}, \mathrm{m}$, $\left.\mathrm{CH}_{2}\right) ;{ }^{13} \mathrm{C}-\mathrm{NMR}: \delta 182.05(\mathrm{C}), 158.07(\mathrm{C}), 152.24(\mathrm{C}), 150.56(\mathrm{C}), 147.02(\mathrm{C}), 133.21(\mathrm{CH}), 132.70(\mathrm{CH})$, 132.25(C), $130.03(\mathrm{CH}), 128.08(\mathrm{CH}), 125.77(\mathrm{C}), 125.48(\mathrm{C}), 119.38(\mathrm{C}), 108.64(\mathrm{C}), 108.53(\mathrm{CH})$, 105.68(C), 61.12( $\left(\mathrm{OCH}_{3}\right), 61.08\left(\mathrm{OCH}_{3}\right), 55.21\left(\mathrm{OCH}_{3}\right), 36.19\left(\mathrm{CH}_{2}\right), 21.67\left(\mathrm{CH}_{2}\right)$. HRMS (ESI-TOF) calcd for $\mathrm{C}_{21} \mathrm{H}_{18} \mathrm{BrNO}_{5}\left(\left[\mathrm{M}+\mathrm{H}^{+}\right]\right)=444.0441$, Found 444.0519 .

1-(2-Bromophenyl)-5,6-dihydro-8,9-methylenedioxypyrrolo[2,1-a]isoquinoline-2,3-dione (10c). In a similar manner, 10c was obtained in $47.7 \%$ yield from ethanol as a deep red prisms; m.p. $226-227^{\circ} \mathrm{C}$; UV (MeOH) $\lambda_{\max } \mathrm{nm}(\log \varepsilon): 203$ (4.64), 236sh (4.18), 261sh (3.88), 284 (3.77), 320 (3.67), 388 (3.64); IR ( $\mathrm{CH}_{2} \mathrm{Cl}_{2}$-film) $v_{\max } \mathrm{cm}^{-1}:$ 3056, 2906, 1744, 1698, 1608, 1568, 1505, 1467, 1403, 1378, 1338, 1316, 1286, 1249, 1181, 1036, 938, 868, 748, 736; ${ }^{1} \mathrm{H}-\mathrm{NMR}: \delta$ 7.71-7.66 (1H, m, Ar-H); 7.42$7.36(1 \mathrm{H}, \mathrm{m}, \mathrm{Ar}-\mathrm{H}) ; 7.31-7.23(2 \mathrm{H}, \mathrm{m}, \mathrm{Ar}-\mathrm{H}) ; 6.79(1 \mathrm{H}, \mathrm{s}, \mathrm{Ar}-\mathrm{H}) ; 6.56(1 \mathrm{H}, \mathrm{s}, \mathrm{Ar}-\mathrm{H}) ; 6.00$ (2H, AB q, $\left.J=1.1 \mathrm{~Hz}, \mathrm{OCH}_{2} \mathrm{O}\right) ; 3.94-3.77\left(2 \mathrm{H}, \mathrm{m}, \mathrm{CH}_{2}\right) ; 3.07\left(2 \mathrm{H}, \mathrm{t}, J=6.3 \mathrm{~Hz}, \mathrm{CH}_{2}\right) ;{ }^{13} \mathrm{C}-\mathrm{NMR}: \delta 181.94(\mathrm{C})$, 158.10(C), 157.94(C), 152.47(C), 147.37(C), 135.17(C), 133.43(CH), 132.43(CH), 131.68(C), 130.13(CH), 128.14(CH), 125.35(C), 118.23(C), 109.28(CH), 108.50(CH), 108.23(C), 102.25( $\left.\mathrm{CH}_{2}\right)$, 36.20 $\left(\mathrm{CH}_{2}\right), 29.24\left(\mathrm{CH}_{2}\right)$. HRMS (ESI-TOF) calcd for $\mathrm{C}_{19} \mathrm{H}_{12} \mathrm{BrNO}_{4}\left(\left[\mathrm{M}+\mathrm{H}^{+}\right]\right)=398.0022$, Found 397.9895 .

Telisatin A (1). A solution of 1,1'-azobis(cyclohexanecarbonitrile) (245.0 $\mathrm{mg}, 1.0 \mathrm{mmol}$ ) and tributyltin hydride $(1.2 \mathrm{~g}, 4.0 \mathrm{mmol})$ in toluene $(20 \mathrm{~mL})$ was added dropwise in four equal portions over $3 \mathrm{~h}$ to a refluxing solution of $10 \mathrm{a}(413.0 \mathrm{mg}, 1.0 \mathrm{mmol})$ in toluene $(20 \mathrm{~mL})$ and the resulting mixture was then refluxed for another $8 \mathrm{~h}$. The solvent was then removed under vacuum and the residue was dissolved in acetonitrile $(40 \mathrm{~mL})$ and washed with hexane $(2 \times 30 \mathrm{~mL})$, then dried over anhydrous sodium sulfate. Removal of the solvent gave a brown viscous oil ( $0.4 \mathrm{~g})$ which was recrystallized with ethanol to give telisatin A (1) as red prisms (109.9 mg, 33.0\%); m.p. 234-235 ${ }^{\circ} \mathrm{C}$ (Lit. [1] m.p. 238-239 ${ }^{\circ} \mathrm{C}$ ); UV (MeOH) $\lambda_{\max } \mathrm{nm}(\log \varepsilon): 207$ (4.03), 257 (4.26), 284sh (3.60), 322 
(3.70), 336 (3.80), 352sh (3.56); IR $\left(\mathrm{CH}_{2} \mathrm{Cl}_{2}\right.$-film) $v_{\max } \mathrm{cm}^{-1}: 2925,1748,1701,1605,1584,1531$, 1462, 1423, 1386, 1306, 1261, 1195, 1149, 1131, 1112, 1037, 969, 924, 802, 759. ${ }^{1} \mathrm{H}-\mathrm{NMR}$ 8: 9.41 $(1 \mathrm{H}$, br d, $J=8.5 \mathrm{~Hz}, \mathrm{H}-11) ; 8.63(1 \mathrm{H}, \mathrm{dd}, J=8.0,1.5 \mathrm{~Hz}, \mathrm{H}-8) ; 7.67-7.60$ (1H, m, H-9); 7.54-7.46 $(1 \mathrm{H}, \mathrm{m}, \mathrm{H}-10) ; 7.17(1 \mathrm{H}, \mathrm{s}, \mathrm{H}-3) ; 4.10\left(3 \mathrm{H}, \mathrm{s}, \mathrm{OCH}_{3}\right) ; 3.97\left(2 \mathrm{H}, \mathrm{t}, J=6.5 \mathrm{~Hz}, \mathrm{CH}_{2}\right) ; 3.95(3 \mathrm{H}, \mathrm{s}$, $\left.\mathrm{OCH}_{3}\right) ; 3.35\left(2 \mathrm{H}, \mathrm{t}, J=6.5 \mathrm{~Hz}, \mathrm{CH}_{2}\right)$; ${ }^{13} \mathrm{C}-\mathrm{NMR}: \delta 179.98(\mathrm{C}), 160.34(\mathrm{C}), 157.15(\mathrm{C}), 153.36(\mathrm{C})$, 146.65(C), $130.75(\mathrm{C}), 129.35(\mathrm{C}), 129.21(\mathrm{CH}), 128.33(\mathrm{CH}), 127.56(\mathrm{C}), 125.87(\mathrm{C}), 125.62(\mathrm{CH})$, 123.76(CH), 112.29(CH), 112.18(C), 103.17(C), 59.99( $\left(\mathrm{OCH}_{3}\right)$, 56.62( $\left(\mathrm{OCH}_{3}\right), 36.53\left(\mathrm{CH}_{2}\right), 27.68$ $\left(\mathrm{CH}_{2}\right)$. HRMS (ESI-TOF) calcd for $\mathrm{C}_{20} \mathrm{H}_{15} \mathrm{NO}_{4}\left(\left[\mathrm{M}+\mathrm{H}^{+}\right]\right)=334.1074$, Found 334.1125.

Telisatin B (2). In a similar manner, telisatin B (2) was obtained as deep red prisms (30.0\%) from ethanol; m.p. $218-219{ }^{\circ} \mathrm{C}$ (Lit.[1] m.p. $221-222{ }^{\circ} \mathrm{C}$ ); UV (MeOH) $\lambda_{\max } \mathrm{nm}(\log \varepsilon): 203$ (4.33), 223sh (4.26), 257 (4.59), 318sh (3.99), 329 (4.07); IR $\left(\mathrm{CH}_{2} \mathrm{Cl}_{2}-\mathrm{film}\right) v_{\max } \mathrm{cm}^{-1}: 2942,2864,1749,1716$, 1702, 1619, 1607, 1579, 1527, 1515, 1452, 1406, 1389, 1323, 1146, 1125, 1071, 1033, 973, 814, 757; ${ }^{1} \mathrm{H}-\mathrm{NMR}: \delta 9.37(1 \mathrm{H}, \mathrm{br} \mathrm{d}, J=8.5 \mathrm{~Hz}, \mathrm{H}-11), 8.60(1 \mathrm{H}, \mathrm{dd}, J=8.0,1.3 \mathrm{~Hz}, \mathrm{H}-8), 7.64-7.56(1 \mathrm{H}, \mathrm{m}$, $\mathrm{H}-9), 7.55-7.45(1 \mathrm{H}, \mathrm{m}, \mathrm{H}-10), 4.14\left(3 \mathrm{H}, \mathrm{s}, \mathrm{OCH}_{3}\right), 4.03\left(3 \mathrm{H}, \mathrm{s}, \mathrm{OCH}_{3}\right), 4.01\left(3 \mathrm{H}, \mathrm{s}, \mathrm{OCH}_{3}\right), 3.91$ $\left(2 \mathrm{H}, \mathrm{t}, J=6.5 \mathrm{~Hz}, \mathrm{CH}_{2}\right), 3.33\left(2 \mathrm{H}, \mathrm{t}, J=6.5 \mathrm{~Hz}, \mathrm{CH}_{2}\right) ;{ }^{13} \mathrm{C}-\mathrm{NMR}: \delta 180.69(\mathrm{C}), 159.86(\mathrm{C}), 152.58(\mathrm{C})$, 152.16(C), 152.03(C), 150.16(C), 128.71(CH), 127.53(CH), 126.63(C), 126.38(C), 126.11(C), 125.78(CH), $123.66(\mathrm{CH}), \quad 121.18(\mathrm{C}), \quad 114.14(\mathrm{C}), \quad 104.17(\mathrm{C}), \quad 61.47\left(\mathrm{OCH}_{3}\right), \quad 61.30\left(\mathrm{OCH}_{3}\right), 60.48$ $\left(\mathrm{OCH}_{3}\right), 36.14\left(\mathrm{CH}_{2}\right), 21.21\left(\mathrm{CH}_{2}\right)$; HRMS (ESI-TOF) calcd for $\mathrm{C}_{21} \mathrm{H}_{17} \mathrm{NO}_{5}\left(\left[\mathrm{M}+\mathrm{H}^{+}\right]\right)=364.1179$, Found 364.1231.

Lettowianthine (3). In a similar manner, lettowianthine (3) was obtained as red prisms (34.0\%); m.p. 294-295 ${ }^{\circ} \mathrm{C}$ (dec.) (Lit. [2] m.p. $314-317{ }^{\circ} \mathrm{C}$ (dec.); Lit.[4] m.p. 265-267 ${ }^{\circ} \mathrm{C}$ ); UV (MeOH) $\lambda_{\max } \mathrm{nm}$ (log $\varepsilon): 203$ (4.37), 212sh (4.29), 247sh (4.07), 257sh (4.04), 287 (3.64), 335 (3.51), 353 (3.28). IR $\left(\mathrm{CH}_{2} \mathrm{Cl}_{2}\right.$-film) $v_{\max } \mathrm{cm}^{-1}: 2923,2093,1737,1695,1622,1610,1581,1530,1506,1450,1417,1301$, 1253, 1222, 1176, 1151, 1122, 1050, 927, 867, 749; ${ }^{1} \mathrm{H}-\mathrm{NMR}: \delta 8.80$ (1H, br d, $\left.J=8.6 \mathrm{~Hz}, \mathrm{H}-11\right)$, $8.55(1 \mathrm{H}, \mathrm{br} \mathrm{d}, J=7.6 \mathrm{~Hz}, \mathrm{H}-8), 7.61(1 \mathrm{H}, \mathrm{t}, J=6.9 \mathrm{~Hz}, \mathrm{H}-9), 7.48(1 \mathrm{H}, \mathrm{t}, J=7.2 \mathrm{~Hz}, \mathrm{H}-10), 7.09$ $(1 \mathrm{H}, \mathrm{s}, \mathrm{H}-3), 6.35\left(2 \mathrm{H}, \mathrm{s}, \mathrm{OCH}_{2} \mathrm{O}\right), 3.93\left(2 \mathrm{H}, \mathrm{t}, J=6.2 \mathrm{~Hz}, \mathrm{CH}_{2}\right), 3.29\left(2 \mathrm{H}, \mathrm{t}, J=6.2 \mathrm{~Hz}, \mathrm{CH}_{2}\right) ;{ }^{13} \mathrm{C}-$ NMR $\delta$ : 179.92(C), 160.33(C), 153.68(C), 151.55(C), 143.08(C), 129.61(C), 129.33(CH), 127.62 $(\mathrm{CH}), 126.83(\mathrm{C}), 125.35(\mathrm{CH}), 124.45(\mathrm{C}), 123.60(\mathrm{CH}), 119.90(\mathrm{C}), 112.46(\mathrm{C}), 109.23(\mathrm{CH}), 103.10$ (C), 102.35( $\left(\mathrm{CH}_{2}\right), 36.66\left(\mathrm{CH}_{2}\right), 27.51\left(\mathrm{CH}_{2}\right)$; HRMS (ESI-TOF) calcd for $\mathrm{C}_{19} \mathrm{H}_{11} \mathrm{NO}_{4}\left(\left[\mathrm{M}+\mathrm{H}^{+}\right]\right)=$ 318.0761, Found 318.0666.

\section{References}

1. Menachery, M.D.; Blake, G.W.; Gourley, R.C.; Freyer, A. Telisatin A, telisatin B, and telitoxinone, three nerw aporphinoids from Telitoxicum peruvianum. J. Nat. Prod. 1995, 58, 1945-1949.

2. Nkunya, M.H.H.; Jonker, S.A.; Makangara, J.J.; Waibel, R.; Achenbach, H. Aporphinoid alkaloids and other constituents from Lettowianthus stellatus. Phytochemistry 2000, 53, 1067 1073. 
3. Chen, C.-C.; Huang, Y.-L.; Lee, S-S.; Ou, J.-C. Laurodionine, a new oxalyl-fused aporphine alkaloid from Phoebe formasana. J. Nat. Prod. 1997, 60, 826-827.

4. Chang, F.-R.; Chen, C.-Y.; Hsieh, T.-J.; Cho, C-P.; Wu, Y.-C. Chemical constituents from Annona glabra III. J. Chin. Chem. Soc. 2000, 47, 913-920.

5. Saa, J.M. ; Cava, M.P. Dehydroaporphines. An acylation study. J. Org. Chem. 1978, 43, 10961099.

6. Castedo, L.; Saa, C.; Saa, J.M.; Suau, R. Synthesis of oxoaporphines. An unusual photocyclization-photoreduction of 2,3-diaryl- $\Delta^{2}$-pyrroline-4,5-diones. J. Org. Chem. 1982, 47, 513-517.

7. Saa, C.; Guitian, E.; Castedo, L.; Suau, R.; Saa, J.M. A regioselective entry to 13-substituted 8oxoprotoberberines. Total synthesis of ( \pm )-corydaline. J. Org. Chem. 1986, 51, 2781-2784.

8. Mikhailovskii, A.G. Reactions of 2,3-dioxopyrrolo[2,1-a]isoquinolines with sodium borohydride and the properties of its products. Chem. Heterocycl. Comp. 1996, 32, 590-595.

9. Kuo, R.-Y.; Wu, C.-C.; Chang, F.-R.; Yeh, J.-L.; Chen, I.-J.; Wu, Y.-C. Antiplatelet activity of synthetic pyrrolo-benzylisoquinolines. Bioorg. Med. Chem. Lett. 2003, 13, 821-823.

10. Harrowven, D.C.; Nunn, M.I.T.; Fenwick, D. R. Radical cyclisations to arenes for the synthesis of phenanthrenes. Tetrahedron Lett. 2002, 43, 3185-3187.

11. Harrowven, D.C.; Nunn, M.I.T.; Fenwick, D. R. [5]Helicenes by iterative radical cyclisations to arenes. Tetrahedron Lett. 2002, 43, 3189-3191.

12. Harrowven, D.C.; Nunn, M.I.T.; Fenwick, D.R. [5]Helicenes by tandem radical cyclisation. Tetrahedron Lett. 2002, 43, 7345-7347.

13. Wang, Y.-C.; Lin, C.-H.; Chen, C.-M.; Liou, J.-P. A concise synthesis of denbinobin. Tetrahedron Lett. 2005, 46, 8103-8104.

14. Abbate, S.; Bazzini, C.; Caronna, T.; Fontana, F.; Gambarotti, C.; Gangemi, F.; Longhi, G.; Mele, A.; Sora, I.N.; Panzeri, W. Monoaza[5]helicenes. Part 2: synthesis, characterisation and theoretical calculations. Tetrahedron 2006, 62, 139-148.

15. Niimi, J. Syntheses of biscoclaurine alkaloids. IV. Synthesis of dl-2,4'-bis(2-methyl-6,7dimethoxy-1,2,3,4-tetrahydro-1-isoquinolylmethyl)diphenyl ether. Yakugaku Zasshi 1960, 80, 123-126.

16. Nimgirawath, S.; Taylor, W.C. Photochemical synthesis of $8 H$-Benzo[g]-1,3-benzodioxolo-[6, 5,4-de]quinolin-8-one (Liriodenine) via 7-methyl-6,7-dihydro-5H-benzo[g]-1,3-benzodioxolo[6,5,4-de]quinoline. Aust. J. Chem. 1983, 36, 1061-1065.

Sample Availability: All stable products reported in this paper are available from the authors.

(C) 2009 by the authors; licensee Molecular Diversity Preservation International, Basel, Switzerland. This article is an open-access article distributed under the terms and conditions of the Creative Commons Attribution license (http://creativecommons.org/licenses/by/3.0/). 